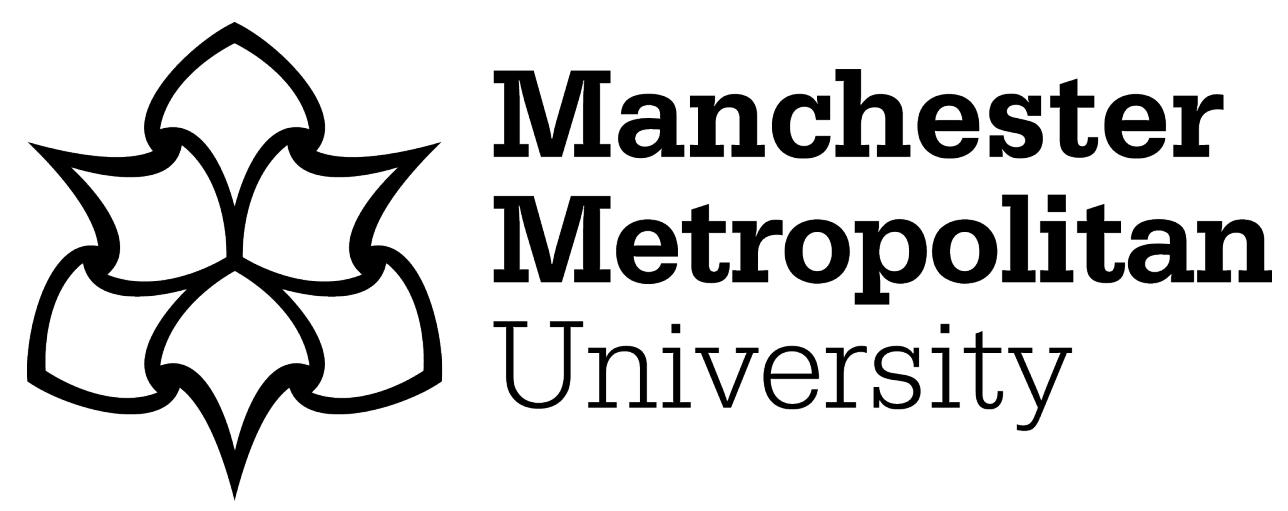

Khait, Anatoliy, Ma, Zhihua ORCID logoORCID: https://orcid.org/0000-00022426-3038, Qian, Ling ORCID logoORCID: https://orcid.org/0000-00029716-2342, Bai, Wei ORCID logoORCID: https://orcid.org/0000-0002-3537207X and Lin, Zaibin (2020) Energy Dissipation and Non-Potential Effects in Wave Breaking. In: The Thirtieth (2020) International Ocean and Polar Engineering Conference, 11 October 2020 - 16 October 2020, Virtual.

Downloaded from: https://e-space.mmu.ac.uk/625423/

Publisher: The International Society of Offshore and Polar Engineer (ISOPE)

Please cite the published version 


\title{
Energy Dissipation and Non-Potential Effects in Wave Breaking
}

\author{
Anatoliy Khait, Zhihua Ma, Ling Qian, Wei Bai and Zaibin Lin \\ Centre for Mathematical Modelling and Flow Analysis, Department of Computing and Mathematics, \\ Manchester Metropolitan University, Chester Street, Manchester M1 5GD, United Kingdom
}

\begin{abstract}
This paper presents a comparative numerical study of the energy dissipation process in the breaking of focused waves by using a potential flow model and a coupled potential/viscous flow model. An empirical eddy viscosity term is introduced to the fully-nonlinear potential (FNP) flow model to account for the breaking wave energy dissipation. The FNP model is further coupled with an incompressible two-phase NavierStokes (NS) flow solver through a one-way linkage to generate and propagate focused waves in the domain. Numerical absorbing regions are placed in front of the outlet boundaries to dampen wave reflection. The standalone FNP model and the coupled FNP+NS model are applied to deal with each scenario comparatively. This enables the accurate quantification and comparison of the energy loss of breaking waves calculated by the two numerical models. The velocity field is decomposed into potential component, which is reconstructed from the corresponding free surface elevation computed in the coupled model by using the weakly-nonlinear wave theory, and non-potential rotational component. Detailed analysis of the numerical results shows that: (1) energy loss is closely related to wave steepness, (2) mild rotational motion produced by a non-breaking wave is local in time with a short life-span, (3) strong non-potential motion triggered by wave breaking is not local in time but persists in the flow for dozens of or even many more wave periods.
\end{abstract}

KEY WORDS: physical oceanography; white capping, eddy viscosity; boundary element method; computational fluid dynamics.

\section{INTRODUCTION}

Wave breaking (white capping) is a transient phenomenon occurring frequently in the propagation of surface gravity water waves in open seas and coastal surf zones. It is of significant importance in coastal and marine engineering due to its capability to produce extreme loadings that can severely damage or completely destroy coastal defence, offshore structure and marine vessel. It also plays an important role in the atmosphere-ocean system by facilitating and enhancing the physical, chemical and biological interactions across the air-sea interface. Breaking is considered as the main sink of the kinematic energy received by ocean waves from winds through complex interactions. It has been shown that wave breaking is also responsible for the momentum, mass and energy exchange between the ocean and the atmosphere (Veron, 2015): it leads to the intensive release of spray into the air, as well as the strong mixing and turbulisation of the upper ocean boundary layer. A recent work of McAllister et al. (2019) shows the significant role played by wave breaking in the formation of rogue waves, which pose a great danger to maritime activities. These aforementioned works demonstrate the importance in the fundamental understanding of wave breaking phenomena.

Investigating the long-term evolution of surface waves in oceans requires the use of large-scale mathematical models that involve a number of assumptions. The most critical assumption is in the flow potentiality of ocean waves, which allows us to deal with the problem either in the physical or in the Fourier space. However, the potential flow assumption fails in the prediction of evolution of highly-nonlinear breaking waves because the flow, in this case, is not irrotational any more. This leads to the need of empirical closures for balancing the energy fluxes. One of the most promising empirical wave breaking closure based on the eddy viscosity approximation was developed recently by Tian et al. (2010, 2012). Although it has been proved to be adequate in the prediction of the integral energy fluxes, there is significant deviation in the free surface profiles produced by this model compared to experimental measurements (Seiffert and Ducrozet, 2018).

Instead using the geometric statistical parameters proposed in the eddy viscosity model of Tian et al. $(2010,2012)$, recent findings of Derakhti et al. (2018) show that the breaking strength might be properly determined from the kinematic characteristics of the wave crest at the inception of breaking. The incorporation of the more advanced kinematic parametrization of the breaking crest may significantly improve the accuracy of the eddy viscosity model. Moreover, the wavelet analysis performed by Derakhti and Kirby (2016) showed that wave breaking causes nonlinear changes in the complex amplitude components, which is neglected in all the existing approaches. Obviously, the lack in fundamental knowledge is considered to be the main reason not allowing improvement of the large-scale ocean models.

This paper aims to improve our fundamental understanding of the nonpotential fluid flows generated by wave breaking. It is expected that a detailed investigation of the evolution and dissipation of the non- 
potential velocity fields will allow us to formulate more accurate semiempirical eddy viscosity approximations for the energy dissipation applicable within the large-scale potential models.

\section{NUMERICAL MODELS}

The problem under investigation requires quantitative comparison of the solution obtained by the fully-nonlinear potential flow model with the results produced by an incompressible two-phase Navier-Stokes flow solver. Taking this into consideration, a boundary element method (BEM) based potential model was coupled with a volume-of-fluid method based incompressible two-phase viscous flow model. In the present paper, the acronym BEM is used to indicate the boundary element method based fully-nonlinear potential flow model, and CFD to represent the incompressible two-phase Navier-Stokes flow model. Schematics of the BEM and CFD domains and their coupling are shown in Fig. 1. The present study is focused on the two-dimensional wave breaking problems.

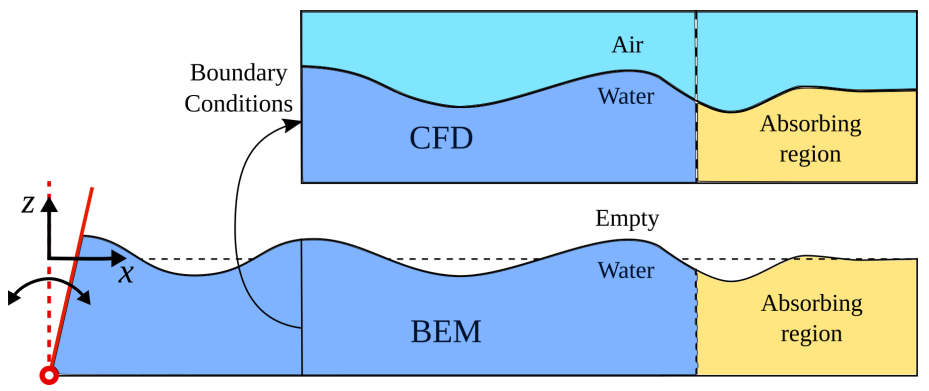

Fig. 1 Schematic of the numerical models coupling

\section{Fully-nonlinear potential model}

Within potential approximation, fluid flow in the interior of the computational domain is explicitly determined by the solution at the domain boundaries by the Greens second identity. This property is used in the BEM method to transform 2D problem into the semi-1D problem defined by the following governing equation:

$\alpha \varphi\left(\mathbf{r}_{\mathbf{s}}\right)=\oint_{\Gamma}\left(\frac{\partial \varphi}{\partial n}(\mathbf{r}) \psi\left(\mathbf{r}, \mathbf{r}_{\mathbf{s}}\right)-\varphi(\mathbf{r}) \frac{\partial \psi}{\partial n}\left(\mathbf{r}, \mathbf{r}_{\mathbf{s}}\right)\right) d \Gamma$

This equation provides values of the flow potential $\varphi$ at the domain boundaries, while the fluid velocity is $\mathbf{U}=-\nabla \varphi$. In the equation, $\psi$ determines the potential flow at point $\mathbf{r}$ due to a source located at $\mathbf{r}_{\mathrm{s}}, \mathbf{r}$ is a radius-vector, $\alpha$ is a constant, $\mathrm{n}$ is the direction normal to the domain boundary. No-penetration condition $\partial \varphi / \partial n=0$ is applied at the bottom and right boundaries of the domain. The kinematic and the dynamic boundary conditions applied at the free surface are given by:

$$
\begin{aligned}
& \frac{D \mathbf{r}}{D t}=-\nabla \varphi \\
& \frac{D \varphi}{D t}=g z-\frac{1}{2}|\nabla \varphi|^{2}-\underbrace{p_{d} \sqrt{g h} \frac{\partial \varphi}{\partial n} b_{f}(x)}_{\text {absorbing region }}+\underbrace{2 v_{\text {eddy }} \frac{\partial^{2} \varphi}{\partial s^{2}}}_{\text {wave breaking }}
\end{aligned}
$$

Here $g$ is the gravitational acceleration, $h$ is the water depth, $p_{d}$ is the dimensionless constant defining the strength of the wave absorption in the numerical absorbing region (see Fig. 1 ), $b_{f}(x)$ is the gradually growing with $x$ function defining location of the absorbing region, $s$ is the direction tangential to the domain boundary. Left boundary of the domain replicates motion of the flap-type wavemaker with a hinge located at the domain bottom.
The breaking of waves generates quite significant rotational flows and, consequently, causes the failure of the potential approximation. Moreover, every breaking event is followed by the loss of continuity of the free surface boundary unallowable in BEM. It was shown by Tian et al. (2010, 2012) that energy dissipation due to wave breaking may be taken into account in the fully-nonlinear potential model by a relatively simple empirical eddy viscosity approximation. The value of the eddy viscosity in (3) was related to the characteristic time- and length-scales (both horizontal and vertical) of the breaking wave: $T_{b r}, L_{b r}$ and $H_{b r}$.

$v_{e d d y}=\chi \frac{H_{b r} L_{b r}}{T_{b r}}$

The proportionality dimensionless constant was found to be $\chi \approx 0.02$. Values of $T_{b r}, L_{b r}$ and $H_{b r}$ are empirically related to geometric characteristics of the wave shape at the breaking inception.

Domain boundaries were discretized using the $3^{\text {rd }}$-order MII method. Several grids having resolution in the range 16-42 nodes per characteristic wave length $\left(\lambda_{0}\right)$ were considered to find the parameters ensuring the grid independent solution. It was found that the resolution 32 nodes per $\lambda_{0}$ or higher resulted in almost identical results. Integration time step was taken to satisfy the numerical stability criterion defined by the Courant number $C F L \leq 0.1$. Details on the numerical grid and the geometry of the domain are summarized in Table 1. Complete description of the numerical schemes is presented in (Khait and Shemer, 2018).

Table 1 Parameters of the BEM computational domain

\begin{tabular}{ll} 
Length & $20 \mathrm{~m}$ \\
Length of the numerical absorbing region & $5 \mathrm{~m}$ \\
Still water depth $h$ & $0.6 \mathrm{~m}$ \\
Number of free surface nodes & 836 \\
Number of nodes at the bottom boundary & 522 \\
Number of nodes at the side boundaries & 26 \\
\hline
\end{tabular}

\section{Two-phase CFD model}

The OpenFOAM ${ }^{\circledR}$ toolset with a modified interFoam solver was used to perform viscous flow simulations. The Volume-of-Fluid (VOF) method was used to describe the transport of the two-phase mixture of air and water with a relatively sharp interface at the free surface. The governing equations represent momentum and mass conservation laws supplemented with the transport equation for the volumetric fraction of water:

$$
\begin{aligned}
& \frac{\partial \rho \mathbf{U}}{\partial t}+\nabla \cdot(\rho \mathbf{U} \mathbf{U})=\nabla \cdot(\mu \nabla \mathbf{U})+\sigma \kappa \nabla \alpha-\mathbf{g} \cdot \mathbf{r} \nabla \rho-\mathbf{p}_{\mathbf{d}} \\
& \nabla \cdot \mathbf{U}=0 \\
& \frac{\partial \alpha}{\partial t}+\nabla \cdot(\alpha \mathbf{U})=0
\end{aligned}
$$

Density of the mixture $\rho$ is determined by the water volumetric fraction $\alpha$ as follows: $\rho=\alpha \rho_{w}+(1-\alpha) \rho_{a}$, where $\rho_{w}$ and $\rho_{a}$ are densities of water and air respectively. Similar expression is used to determine the dynamic viscosity of the mixture $\mu$. Equation (5) involves the dynamic pressure $\mathbf{p}_{\mathbf{d}}=\mathbf{p}-\rho \mathbf{g} \cdot \mathbf{r}$. Surface tension force is taken into account by the coefficient $\sigma$ and the local interface curvature $\kappa$. Because of large difference between $\rho_{w}$ and $\rho_{a}$, VOF method is to be complement by the artificial compression of $\alpha$ near the regions of its high gradients in order to maintain sharp air-water interface. Variation of pressure $p_{d}$ in time is computed iteratively using the implicit PISO method.

The pseudo-2D domain was discretized by rectangular cuboid mesh cells, with a one layer of mesh cell in $y$ direction only (see Fig. 1). The 
Reynolds number in this case can be estimated as:

$\operatorname{Re}=\frac{\rho_{w} g^{1 / 2} \lambda^{3 / 2}}{\mu_{w}} \approx 2 \times 10^{5}$

Even for a two-dimensional problem, numerical simulation of flows at such high $R e$ would require enormous computational effort to resolve all scales appearing as a result of the wave breaking. However, the dominant flow contribution is introduced by the underlying surface water wave, which is assumed to be potential and laminar. Therefore, it is expected to have the grid convergence in terms of the free surface elevation. Several meshes having different grid resolution were considered to examine the grid independence of the numerical solution. It was established that the mesh having 256 cells per characteristic wave length $\left(\lambda_{0}\right)$ provides the converging solution while balancing the computational efficiency and the resolving of the small scale flow features. The total number of cells used in the present CFD simulation is $N \approx 1.9 \times 10^{6}$. Waves reflected from the far-end boundary of the domain are dampened in the numerical absorbing region utilizing the effective viscosity to dissipate the flow velocities. According to the recommendations for the interFoam solver, the computational stability is achieved when $C F L \leq 0.5$ (Larsen et al., 2019). Thus the integration time step was selected to be adaptive maintaining this stability criterion. Time integration was performed using the implicit Euler scheme. Details on the domain configuration are summarized in Table 2.

Table 2 Parameters of the CFD computational domain

\begin{tabular}{ll} 
Coordinate of the left boundary & $3.0 \mathrm{~m}$ \\
Length & $16.8 \mathrm{~m}$ \\
Length of the numerical absorbing region & $1.5 \mathrm{~m}$ \\
Still water depth & $0.6 \mathrm{~m}$ \\
Total height of the domain & $1.2 \mathrm{~m}$ \\
Number of grid cell in the horizontal direction & 4608 \\
Number of grid cell in the vertical direction & 400 \\
\hline
\end{tabular}

\section{Coupling of the models}

For the purpose of the present study a one-way coupling was considered when the solution obtained in the BEM model is transferred to the CFD model. The coupling of BEM and CFD models is achieved by definition of the corresponding initial and boundary conditions. Coordinates of the cell centres of the CFD mesh were used in the BEM model to calculate the interior velocity field $\mathbf{U}$ at the instant of the CFD model initialization. In turn, the needed velocity field $\mathbf{U} \equiv\{u, v, w\}=-\nabla \varphi$ should be estimated numerically from the field of the flow potential $\varphi$ available in the BEM model. Several techniques were examined in terms of the accuracy and the computational efficiency. It was found that the simple central differencing scheme provides a reasonable accuracy, while keeping the process computationally efficient:

$u=-\frac{\varphi(x+\Delta x, z)-\varphi(x-\Delta x, z)}{2 \Delta x}$

$w=-\frac{\varphi(x, z+\Delta z)-\varphi(x, z-\Delta z)}{2 \Delta z}$

Here the resolution of the scheme $\Delta x=\Delta z$ was taken equal to $1 / 10$ of the size of the CFD mesh cell (also $1 / 6$ and $1 / 16$ values were examined). The values of the potentials $\varphi(x, z)$ in (9) and (10) were calculated in the BEM solver by selecting the location of the source $\mathbf{r}_{\mathbf{s}}=(x, z)$ at the given cell centre coordinates $(x, z)$ and performing integration of (1).

Similar procedure was done in the BEM model to extract the variation with time of the fluid velocities $\mathbf{U}$ at the location corresponding to the position of the left boundary of the CFD domain in order to determine the corresponding boundary condition. Since active wave breaking resulted in the deviation of the wave shapes in BEM and CFD models, as will be shown consequently, coupling of the models at the right boundary is not considered in the present work.

\section{Wave generation}

Post-processing of the BEM and CFD simulation results is performed involving the spatial Fourier transform. Avoiding side effects due to imperfect spatial periodicity suggests utilization of the broad-banded focusing wave train. The most active wave breaking appears at the focusing location near the centre of the domain, when waves are almost absent at the sides of the domain leading to spatial periodicity of the surface elevation. The wave train having Gaussian-shaped envelope at the focusing location is:

$\eta(t)_{x_{f}}=\zeta_{0} \exp \left\{-\left(\frac{t}{m T_{0}}\right)^{2}\right\} \cos \left(\omega_{0} t\right)$,

where parameter $m=0.6$ for broad-banded wave train, $T_{0}=0.7 \mathrm{~s}$ and $\omega_{0}=2 \pi / T_{0}$ are the central wave period and angular frequency. According to the linear dispersion relation $\omega_{0}^{2}=g k_{0} \tanh \left(k_{0} h\right)$, the central wave length and wave number are $\lambda_{0}=0.765 \mathrm{~m}$ and $k_{0}=2 \pi / \lambda_{0}$. Dimensionless water depth corresponds to deep water condition $k_{0} h=4.93>\pi$ (Dean and Dalrymple, 1991). The location of the linear focus of the wave train relative to the wave generating boundary in the BEM domain $(x=0)$ was selected as $x_{f}=8.5 \mathrm{~m}$.

To generate the wave train, a simple linear theory was applied to recalculate the surface elevation given at $x_{f}$ to the motion of the flap-type wavemaker. The wave shape given by Eq. (11) was decomposed into the discrete harmonic components:

$\eta(t, x)=\operatorname{Re}\left\{\sum_{j} a_{j} \exp \left[i\left(k_{j} x-k_{j} x_{f}-\omega_{j} t\right)\right]\right\}$

Thus according to Eq. (12), surface elevation at the wavemaker location is $\eta(t, x=0)$. Linear transfer function (Dean and Dalrymple, 1991) was further applied to recalculate of the surface elevation into the motion of the wavemaker.

Wave breaking intensity was regulated by the constant $\zeta_{0}$ in Eq. (11). Six cases were investigated: $k_{0} \zeta_{0}=0.2,0.3,0.4,0.6,0.8$ and 1.0. It is known that waves break when their steepness satisfy the condition $k_{0} \zeta_{0}>0.3-0.4$. Increase of the wave steepness of the linearly focused wave train beyond these values leads to the single or multiple breaking events of different intensity in both BEM and CFD models.

\section{RESULTS AND DISCUSSION}

\section{Surface elevation}

Spatial and temporal variation of surface elevation was recorded in both BEM and CFD models. In contrast to the BEM model where the surface elevation is explicitly given by the shape of the upper boundary, in CFD model determination of the free surface shape is not straightforward because of the diffused interface between water and air phases. In this paper it is assumed that the free surface corresponds to $\alpha=0.5$ and may be found using iterative interpolation technique. Application of the discrete Fourier transform imposes requirements to the spatial and temporal resolution of the surface elevation records. Temporal resolution was chosen to be equal to the integration time step. Distribution of the surface elevation in space was recorded within the 
(a)

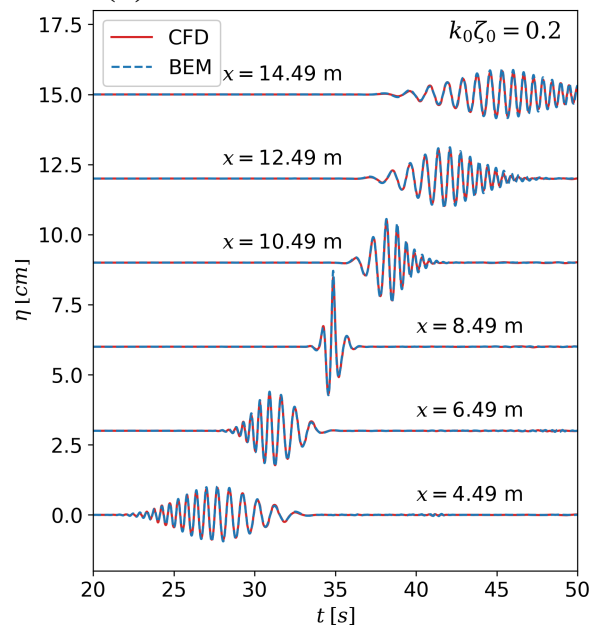

(d)

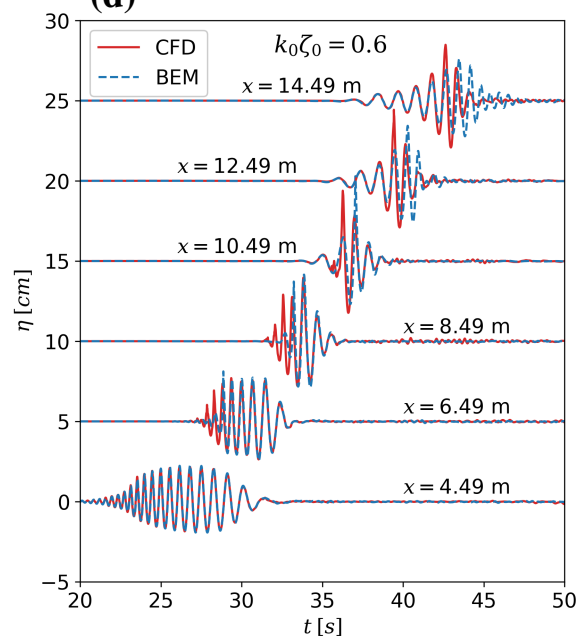

(b)

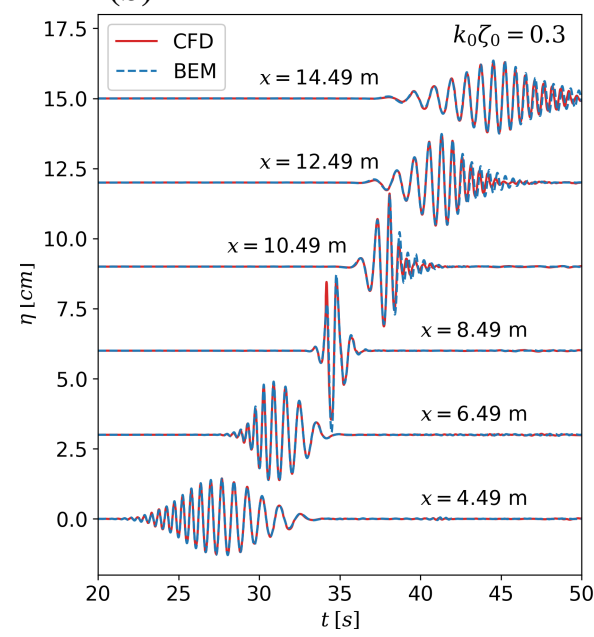

(e)

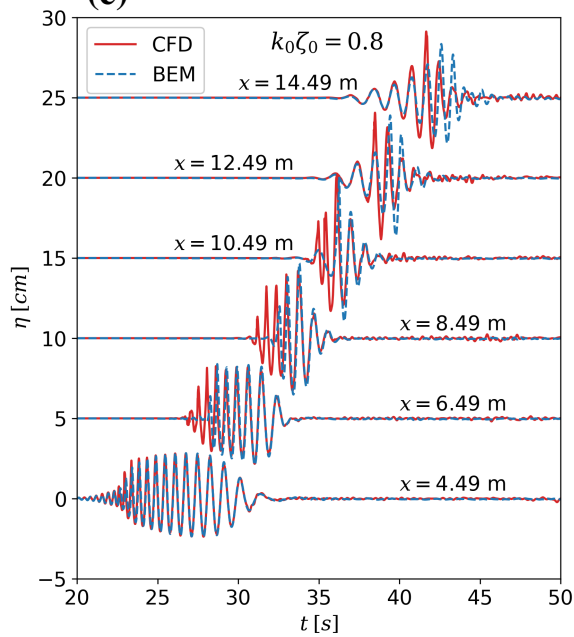

(c)

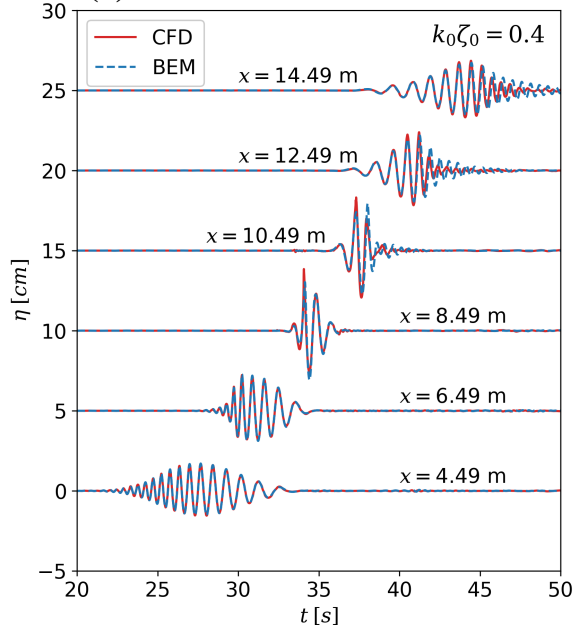

(f)

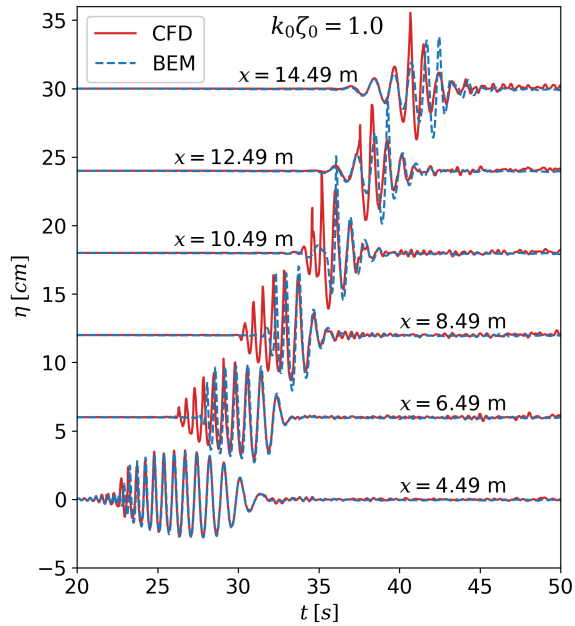

Fig. 2 Surface elevation records at several locations in BEM and CFD domains

interval $x=3.1-15.34 \mathrm{~m}$ having length equal to $16 \lambda_{0}$. Records were obtained at 2048 equidistantly distributed points.

Fig. 2 shows surface elevation probed at six locations for wave trains having different target steepness $k_{0} \zeta_{0}$. Moderately steep wave train, panel (a), demonstrates a perfect match between the results of BEM and CFD simulations providing evidence of the models validity. The shape of the surface elevation at the focus location $(x=8.5 \mathrm{~m})$ is not ideally symmetric because of its deviation from the linear solution appearing during the nonlinear wave train evolution. The increase of $k_{0} \zeta_{0}$ leads to weak spilling wave breaking when $k_{0} \zeta_{0}=0.3$ and multiple stronger plunging breakers for $k_{0} \zeta_{0}=1.0$. It is clearly seen from Fig. 2 (b-f) that intensification of the wave breaking results in the growth of the deviation of the fully-nonlinear potential model results from the CFD results. Obviously, the reason for the given deviation in the surface elevation lies in the insufficient accuracy of the empirical eddy viscosity model used in equation (3). It should be noted that similar deviation of the wave train shape from the experimental measurements was reported in (Tian et al., 2012; Seiffert and Ducrozet, 2018).

\section{Energy dissipation due to wave breaking}

The energy dissipation process during the wave breaking can be investigated by consideration of the energy transferred by the wave train through the different cross sections of the domains. Taking into account that the wave breaking is strongly localized phenomenon, the energy losses are associated with a particular location and can be seen as a reduction of the wave train energy before and after the breaking location.

Nonlinear energy flux at a particular location in the domain is

$F^{N L}(x, t)=\int_{-h}^{\eta(x, t)}\left\{\frac{1}{2} \rho|\mathbf{U}|^{2}+\rho g z+p\right\} u \alpha d z$,

where $\mathbf{U}=(u, v, w)$. Measurement of the nonlinear flux (13) is not feasible in experiments, thus linearisation is usually applied (Tian et al., 2010, 2012; Seiffert and Ducrozet, 2018). For potential linear waves it can be shown that

$F^{L}(x, t) \approx \rho g C_{g s} \eta^{2}(x, t)$,

$C_{g s}=\frac{\sum_{j} C_{g, j} a_{j}^{2}}{\sum_{j} a_{j}^{2}}$ 
(a)

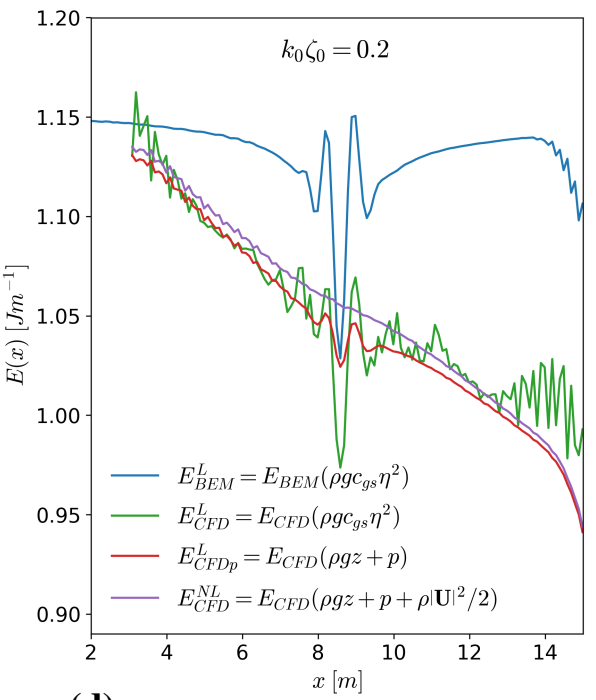

(d)

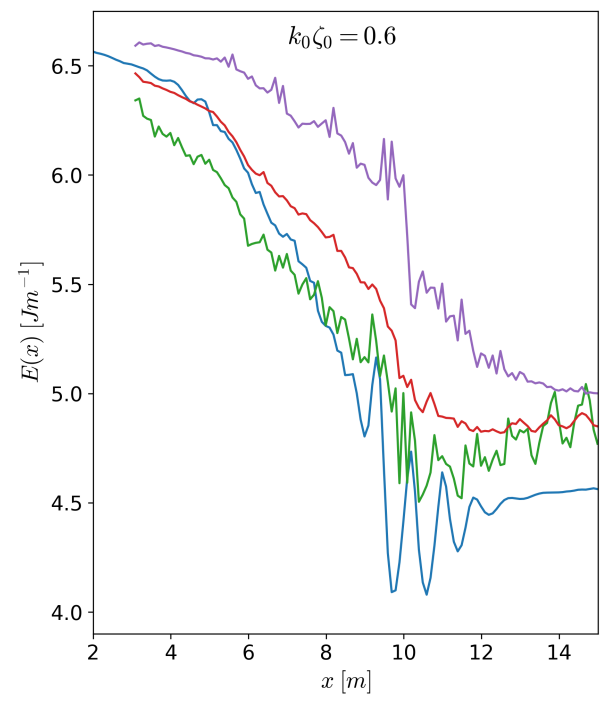

(b)

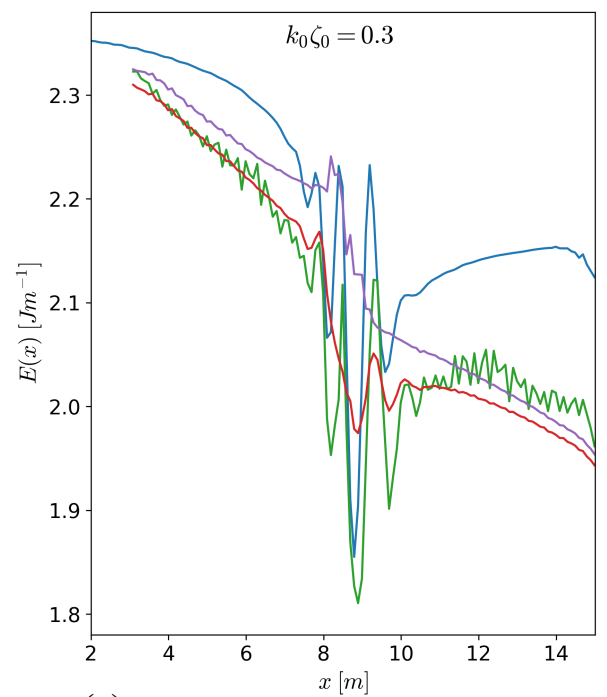

(e)

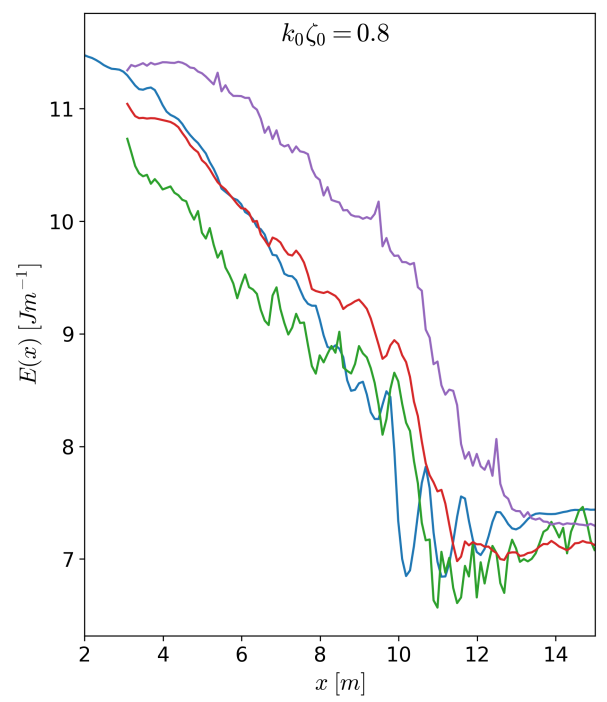

(c)

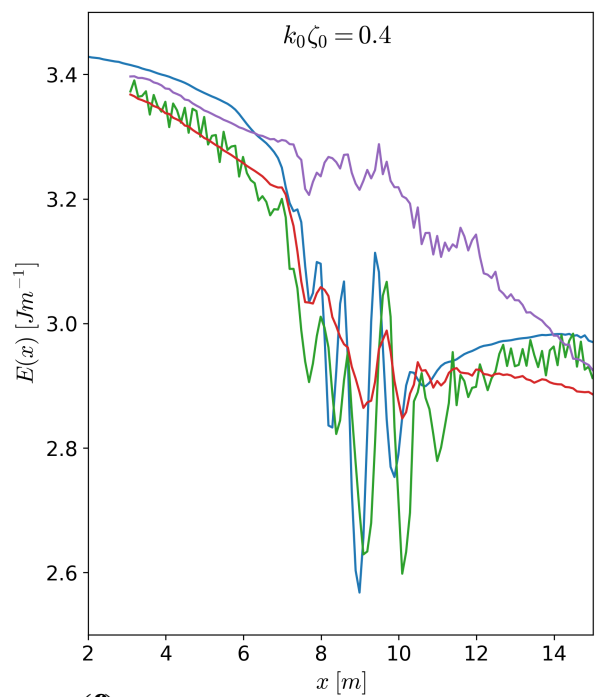

(f)

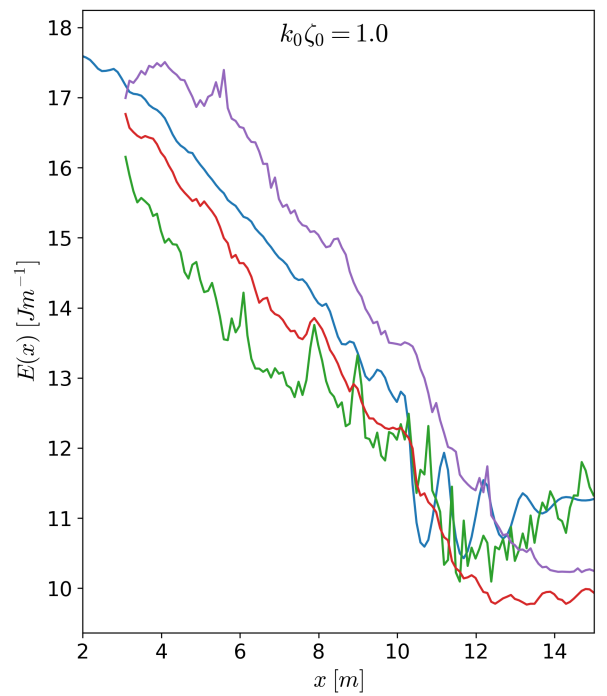

Fig. 3 Integral energy fluxes as a function of the distance from the wavemaker

where $C_{g s}$ is the spectral-weighted group velocity, $a_{j}$ is the amplitude of the $j^{\text {th }}$ harmonic in the Fourier series (12) (Tian et al., 2010). Integration of flux (13) or (14) with time gives the total amount of the energy transferred by the wave train through the particular cross section:

$E(x)=\int_{-\infty}^{\infty} F(x, t) d t$

The advantage of the developed coupled BEM and CFD model was exploited to investigate the applicability of the linear approximation for the energy flux (14). To this end, four different expressions were used to calculate $E(x)$ from the results of BEM and CFD simulations, see Fig. 3. BEM results for moderate steep wave train, $E_{L}^{B E M}$ in Fig. 3(a), demonstrate conservation of energy along the computational domain except for the region close to the absorbing region. Near the focusing location $\left(x_{f}=8.5 \mathrm{~m}\right)$ the waves have the highest steepness, thus the nonlinear effects are the most pronounced. This explains the oscillations of the linear approximation for the energy flux $E_{L}^{B E M}$ near $x_{f}$. Slight decay of the energy with $x$ for the CFD simulations is explained by the viscous dissipation not presented in the irrotational BEM model. A certain contribution to the energy decay in the CFD simulation can be caused by the numerical dissipation of the two-phase flow solver
interFoam. This is minimised in the present study by using a relatively fine mesh with 1.9 million cells, providing adequate numerical accuracy and affordable CPU time cost.

Increase of $k_{0} \zeta_{0}$ (b-f) leads to the appearance of the wave breaking as was discussed above, Fig. 2. It is seen that in all plots the linear approximations for the integral energy flux, i.e. $E_{B E M}^{L}, E_{C F D}^{L}$ and $E_{C F D p}^{L}$, are very close to each other. This highlights that eddy viscosity empirical model for the wave breaking (4) provides a very good prediction for the integral energy dissipation, in agreement with (Tian et al., 2010, 2012; Seiffert and Ducrozet, 2018). On the other hand, the exact nonlinear expression for the energy flux (13) gives higher values of $E_{C F D}^{N L}$ as compared to all liner approximations. This can be explained by the fact that the kinetic energy of the water wave is not dissipated immediately after the breaking, but just transformed into the energy of the rotational fluid flow not carried by the wave train. Such rotational motion contributes to the energy flux, but only near the breaking location, where it is actually present. Since the vortical motion generated by the breaking is not carried by the wave (Melville et al., 2002), all four estimations for the integral energy flux coincide at the far-end of the computational domains for all considered cases. 


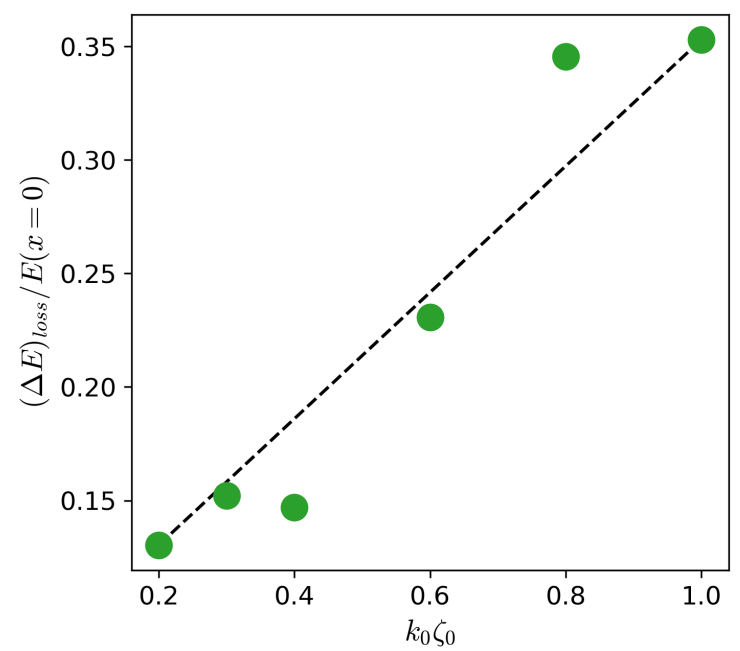

Fig. 4 Total energy loss due to wave breaking of different strength

The normalized total energy loss within the region of the wave breaking can be estimated as $\Delta E_{\text {loss }} / E_{C F D}^{N L}(x=0)$, where $\Delta E_{\text {loss }}=$ $E_{C F D}^{N L}(x=15.3 \mathrm{~m})-E_{C F D}^{N L}(x=3.1 \mathrm{~m})$. It was found that this value is approximately linearly dependant on the maximum steepness of the wave train expected according to the linear theory, i.e. $\Delta E_{\text {loss }} / E_{C F D}^{N L}(x=0) \sim k_{0} \zeta_{0}$, see Fig. 4.

\section{Potential and non-potential components of the flow}

During wave breaking, the energy of the potential flow associated with the surface water waves is partially transformed into the rotational nonpotential flow and usually referred as the energy loss. Quantitative analysis of this process may be achieved by distinguishing the potential $\mathbf{U}_{\mathbf{p}}$ and non-potential $\mathbf{U}_{\mathbf{n p}}$ components of the velocity field obtained in the CFD simulations:

$\mathbf{U}_{\mathbf{C F D}}=\mathbf{U}_{\mathbf{p}}+\mathbf{U}_{\mathbf{n p}}$

The value of $\mathbf{U}_{\mathbf{n p}}$ can be computed by extraction of the results of the BEM simulation from the corresponding results of CFD simulation. However, the significant deviation of the surface elevation shown in Fig. 2 will lead to the physically incorrect results. Therefore, the weakly-nonlinear theory of water waves was used to reconstruct the potential velocity field corresponding to the spatial surface elevation obtained in the CFD simulations.

At the first step, the fully-nonlinear wave number spectrum of the surface elevation (12) was decomposed into the free and the bound waves using the iterative technique based on Zakharov model (Khait and Shemer, 2019):

$\eta(x)=\eta^{(1)}+\eta^{(2)}+O\left(\epsilon^{3}\right)$,

where $\epsilon$ is the measure of the nonlinearity equal to the wave steepness; $\eta^{(1)}$ and $\eta^{(2)}$ is the surface elevation due to free and bound waves respectively. At the second step, surface elevation (18) was recalculated into the velocity fields associated with both free and bound waves:

$$
\begin{aligned}
u_{p}(x, z)= & \operatorname{Re}\left\{\sum_{n}^{N} \Lambda_{n}^{(1)} \cosh \left(k_{n}^{(1)}(h+z)\right) \eta_{n}^{(1)}(x)\right. \\
& \left.+\sum_{m}^{M} \Lambda_{m}^{(2)} \cosh \left(k_{m}^{(2)}(h+z)\right) \eta_{m}^{(2)}(x)\right\}+O\left(\epsilon^{3}\right) \\
w_{p}(x, z)= & \operatorname{Re}\left\{-i \sum_{n}^{N} \Lambda_{n}^{(1)} \sinh \left(k_{n}^{(1)}(h+z)\right) \eta_{n}^{(1)}(x)\right. \\
& \left.-i \sum_{m}^{M} \Lambda_{m}^{(2)} \sinh \left(k_{m}^{(2)}(h+z)\right) \eta_{m}^{(2)}(x)\right\}+O\left(\epsilon^{3}\right)
\end{aligned}
$$

Here $u_{p}$ and $w_{p}$ are the $x$ and $z$ components of the vector $\mathbf{U}_{\mathbf{p}}$ incorporating contributions from both free and bound waves; $N$ and $M$ are the number for the harmonic components in the spectrum defining free and bound waves respectively; $k^{(1)}$ and $k^{(2)}$ are the wave numbers of the free and bound waves; $\Lambda^{(1)}$ and $\Lambda^{(2)}$ are constants. For details see (Khait and Shemer, 2019). Subtraction of of the potential velocity field $\mathbf{U}_{\mathbf{p}}$ from the results of the CFD computations determines the non-potential part of the flow: $\mathbf{U}_{\mathbf{n p}}=\mathbf{U}_{\mathbf{C F D}}-\mathbf{U}_{\mathbf{p}}$.

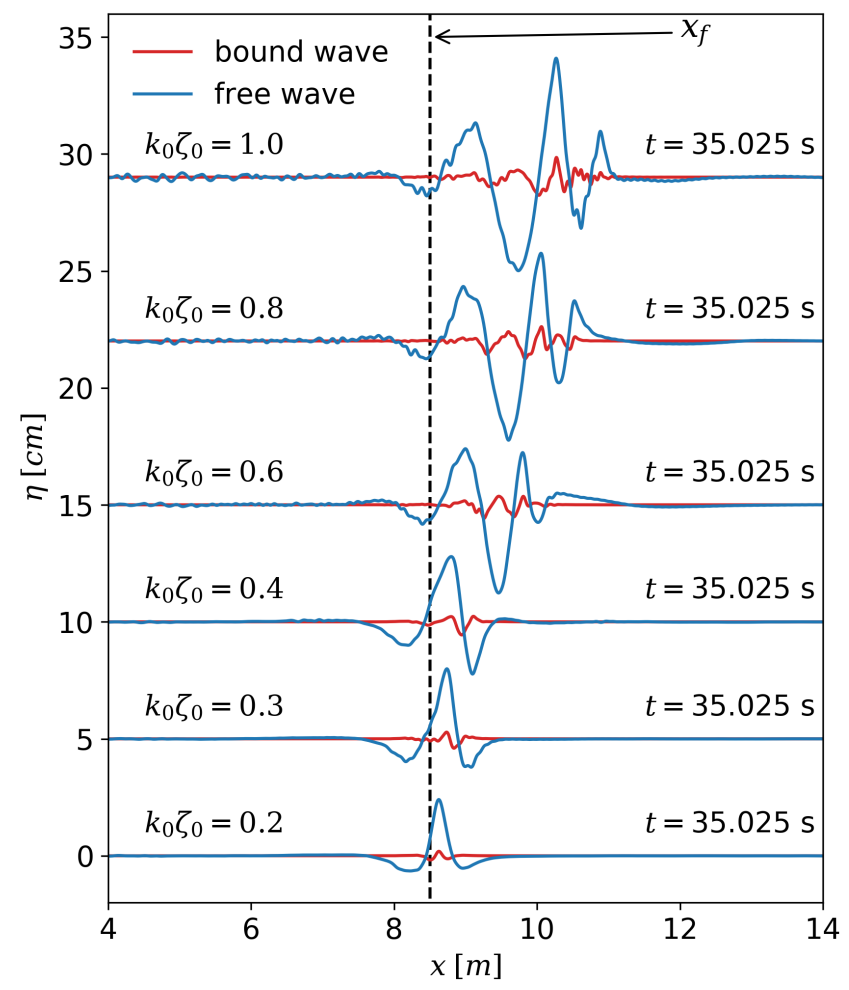

Fig. 5 Decomposition of the surface elevation obtained in the CFD simulations into the free and the bound waves

To verify the correctness of the decomposition (18), the spatial variation of the surface elevation corresponding to both free and bound waves is examined in Fig. 5. Wave trains of different steepness are plotted at the instant of linear focusing $t=35 \mathrm{~s}$. It is seen that the increase of the parameter $k_{0} \zeta_{0}$ leads to the stronger wave nonlinearity, as well as to the intensification of the breaking, finally resulting in the deviation of the shape of the wave train from the expected focused wave.

\section{Generation and evolution of non-potential energy}

To investigate the non-potential effects appearing as a result of the wave breaking, the time-space evolution of the depth-integrated kinetic energy 
(a)

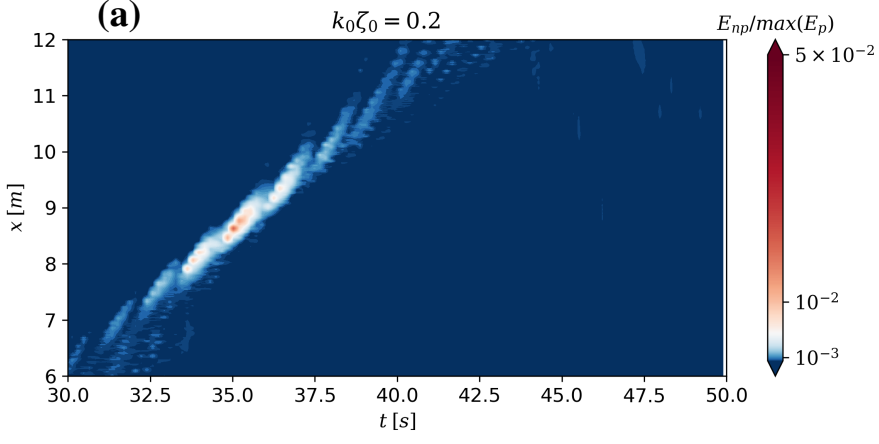

(c)

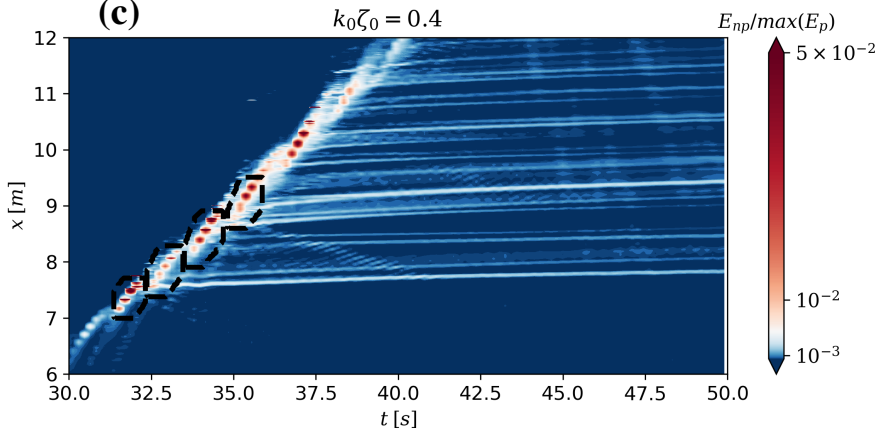

(e)

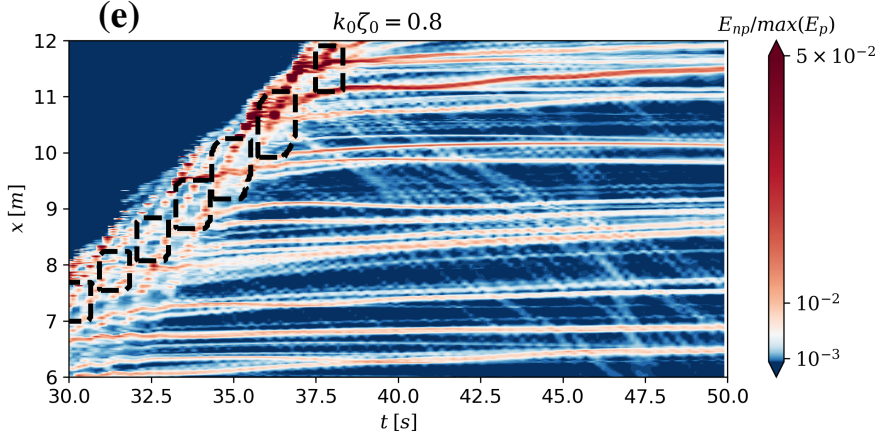

(b)

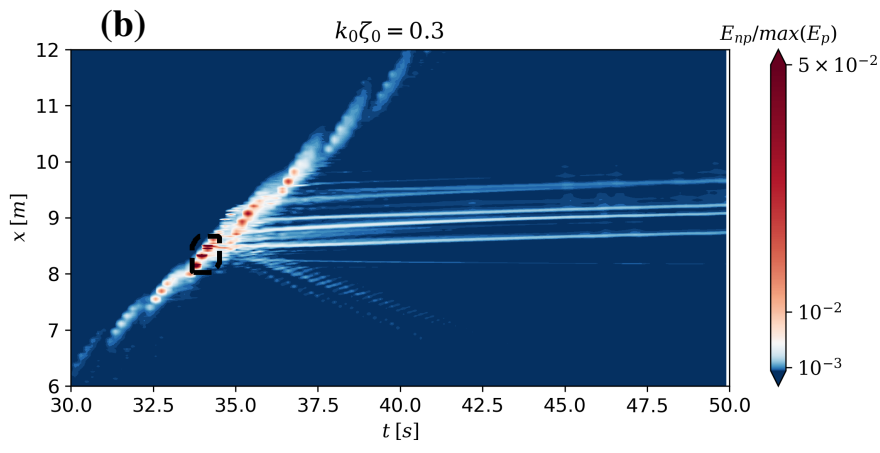

(d)

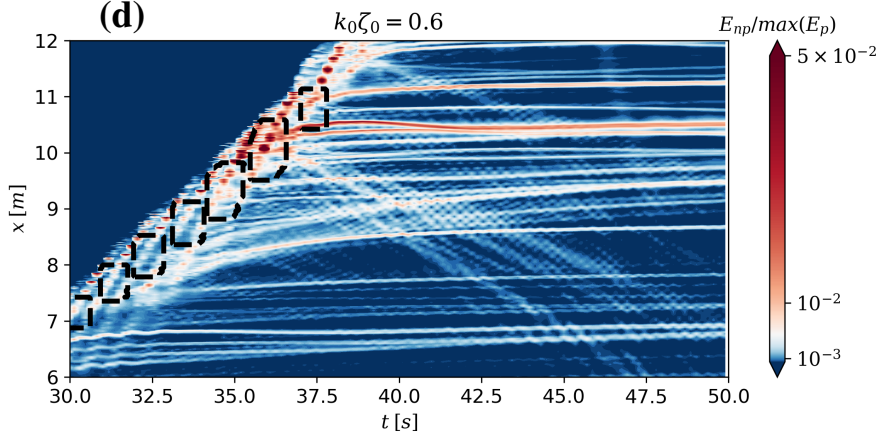

(f)

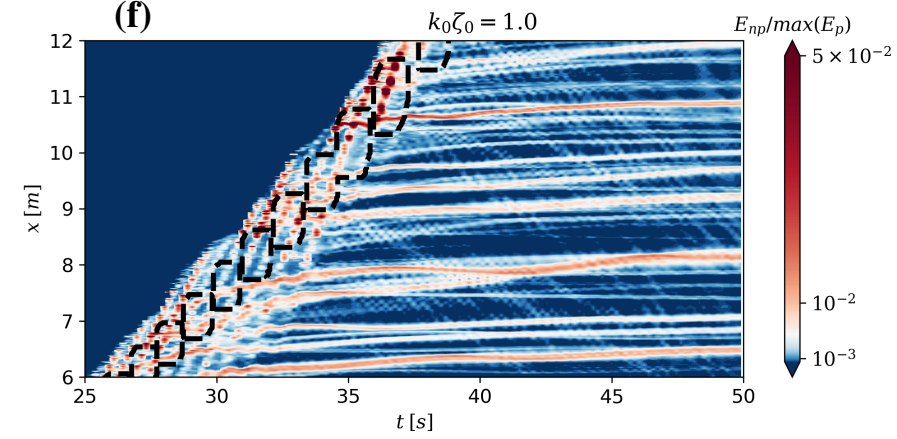

Fig. 6 Space-time diagrams of the depth-integrated non-potential kinetic energy. Black dashed lines depict the regions of the energy dissipation due to the wave breaking approximated by the the eddy viscosity model (4)

associated with the non-potential flow $\mathbf{U}_{\mathbf{n p}}$ (17) was considered:

$$
\begin{aligned}
& E_{n p}(t, x)=\int_{-\infty}^{\infty} d y \int_{-h}^{h} \frac{1}{2} \rho\left|U_{n p}\right|^{2} \alpha d z \\
& E_{p}(t, x)=\int_{-\infty}^{\infty} d y \int_{-h}^{h} \frac{1}{2} \rho\left|U_{p}\right|^{2} \alpha d z
\end{aligned}
$$

Space-time diagrams of the normalized values of the non-potential energy $E_{n p} / \max \left(E_{p}\right)$ are presented in Fig. 6.

In the case of the weakly steep waves with $k_{0} \zeta_{0}=0.2$ when no breaking appears, the distribution of $E_{n p}$ is localized only in the focusing region where the steepest waves are observed. Appearance of $E_{n p}$ is caused by the small deviation of the velocity field near the tip of the steep wave calculated using the weakly-nonlinear approach $(19,20)$ from the results of the viscous two-phase CFD model. At later stages, $E_{n p}$ disappears immediately. The increase of the wave steepness, shown in Fig. 6(b), leads to appearance of several traces of the non-potential energy originating near the focusing location and conserving up to the end of the computation. Note that the positions of the visually observed breaking events coincide with the high values of $E_{n p}$. Interestingly, the non-potential energy generated as a result of the wave breaking is not smoothly distributed in space, but is concentrated in a discrete number of traces (approximately four traces when $k_{0} \zeta_{0}=0.3$ ), each of them represent the stable vortical structure. It can also be seen that the given non-potential structures are slowly moving in the direction of the wave train propagation even when the waves are completely absorbed by the numerical absorbing region at a later time. This indicates that such motion of the vortical structures is not caused by Stokes drift. Moreover, it can be seen that several traces propagate upstream in the domain, opposite to the wave train direction.

Intensification of the wave breaking, see Figs. 6(c-d), is seen as a growth of the number of traces, as well as increase of the value of $E_{n p}$. Trajectories of the traces become more complicated, deviate from the linear shape and demonstrate the varying with time curvature. It is well accepted that the energy dissipation due to the wave breaking may last during several characteristic wave periods. In our approach, the given energy dissipation is the process of transformation of the potential energy $E_{p}$ into the non-potential one $E_{n p}$, which may constitute $E_{n p} / \max \left(E_{p}\right)>5 \%$. It is critical to note that generated non-potential energy $E_{n p}$ does not disappear immediately but can be conserved for several tens or hundreds of characteristic wave periods. Presence of such non-potential motion in the fluid may significantly influence the nonlinear evolution of waves passing the area where the preceding 
breaking was observed.

Black dashed lines in Figs. 6 depict the wave breaking locations where the value of the eddy viscosity $v_{e d d y}>0$ was applied according to the the empirical model (4) in the BEM computations. The energy dissipation areas prescribed by the eddy viscosity are strongly localized in both time and space. Taking into account that the total energy loss by the wave train is accurately predicted in Fig. 3, the transformation of $E_{p}$ into $E_{n p}$ is actually a very fast process. In cases of moderate wave steepness, the eddy viscosity regions coincide with the location of non-potential energy production in CFD model. However, when the wave steepness increases, the eddy viscosity model detects the wave breaking at the locations where CFD model shows absence of the non-potential energy production. This can be explained by the gradual deviation of the wave train shape computed by BEM model from the CFD results. In long-term computation, such inaccuracies may lead to high errors even in the energy losses value.

\section{CONCLUSIONS}

Wave breaking is a two-phase flow phenomenon of significant importance in coastal and marine engineering, and physical oceanography. It causes strong mixing of air and water, and generates turbulence extremely fast. Such a phenomenon needs to be accurately simulated by high-fidelity Navier-Stokes equations based CFD methods. But the numerical solution of two-phase Navier-Stokes equations is highly demanding on the computational resources, which limits its applicability to small-scale problems only. The assumption of flow potentiality simplifies the problem substantially, allowing consideration of the surface water waves evolution at larger scales. It can be complemented, by applying empirical approximations, to take into account the energy dissipation caused by wave breaking. But the application of empirical closures, which ignores underlying important physics, may result in numerical results deviant from high-fidelity CFD calculations and actual physical solutions.

In this paper a coupled potential and two-phase numerical model is proposed to investigate the energy dissipation and non-potential effects in wave breaking. The velocity field obtained by the two-phase model was decomposed into potential and non-potential rotational contributions using the weakly-nonlinear expressions for reconstruction of the velocity field corresponding to the certain shape of the free surface. This approach provides a new insight into the energy evolution process in wave breaking. The process actually involves an energy cascade from the main potential flow to local non-potential rotational flow. The loss of wave energy during breaking is a result of the transformation of potential flow into non-potential one. Detailed analysis of the potential and two-phase numerical results shows that: (1) energy loss is closely related to wave steepness, (2) mild rotational motion produced by a non-breaking wave is local in time with a short life-span, (3) strong non-potential motion triggered by wave breaking is not local in time but persists in the flow for dozens of or even many more wave periods.

It is also found that the eddy viscosity wave breaking approximation applied within the fully-nonlinear potential model allows accurate quantification of the wave energy loss during the breaking events. But it causes the distortion of wave profiles as compared with high-fidelity two-phase computations. The release of the non-potential energy within the eddy viscosity approximation is considered as an immediate loss. On the contrary, two-phase simulations show that the energy of the non-potential flow may significantly contribute to the overall velocity field and can be conserved for dozens (or even hundreds) of characteristic wave periods. This shows that an improvement of the eddy viscosity wave breaking ap- proximation is much needed to take into account the transient characteristics of the non-potential energy released during the breaking events. It may then improve the accuracy of the fully-nonlinear potential solutions.

\section{REFERENCES}

Dean, R.G. and Dalrymple, R.A. (1991). "Water Wave Mechanics for Engineers and Scientists", World Scientific, Singapore.

Derakhti, M., and Kirby, J.T. (2016). "Breaking-onset, energy and momentum flux in unsteady focused wave packets", J. Fluid Mech., vol. 790, pp. 553-581.

Derakhti, M., Banner, M.L., and Kirby, J.T. (2018). "Predicting the breaking strength of gravity water waves in deep and intermediate depth", J. Fluid Mech., vol. 848, R2.

Khait, A. and Shemer, L. (2018). "On the kinematic criterion for the inception of breaking in surface gravity waves: Fully nonlinear numerical simulations and experimental verification", Phys. Fl., 30, 057103.

Khait, A. and Shemer, L. (2019). "Nonlinear wave generation by a wavemaker in deep to intermediate water depth”, Ocean Eng, 182, 222234.

Larsen, B.E., Fuhrman, D.R., Roenby, J. (2019). "Performance of interFoam on the simulation of progressive waves", Coast Eng J, 61:3, 380-400.

Martínez Ferrer, P.J., Causon, D.M., Qian, L., Mingham, C.G., and Ma, Z.H. (2016). "A multi-region coupling scheme for compressible and incompressible flow solvers for two-phase flow in a numerical wave tank", Comput Fluids, 125, 116-129.

McAllister, M.L., Draycott, S., Adcock, T.A.A., Taylor, P.H., van den Bremer, T.S. (2019). "Laboratory recreation of the Draupner wave and the role of breaking in crossing seas", J. Fluid Mech., vol. 860, pp. 767786.

Melville, W.K., Veron, F., and White, C.J. (2002). "The velocity field under breaking waves: coherent structures and turbulence", J. Fl. Mech., 454, 203-233.

Seiffert, B.R., and Ducrozet, G. (2018). "Simulation of breaking waves using the high-order spectral method with laboratory experiments: wave-breaking energy dissipation", Ocean Dynamics, vol. 68, 65-89.

Tian, Z., Perlin, M., and Choi, W. (2010). "Energy dissipation in two-dimensional unsteady plunging breakers and an eddy viscosity model", J. Fl. Mech., 655, 217-257.

Tian, Z., Perlin, M., and Choi, W. (2012). "An eddy viscosity model for two-dimensional breaking waves and its validation with laboratory experiments", Phys. Fl., 24, 036601.

Veron, F. (2015). "Ocean Spray”, Annu. Rev. Fluid Mech., 47:507-38. 